\title{
Making ward-based outreach teams an effective component of human immunodeficiency virus programmes in South Africa
}

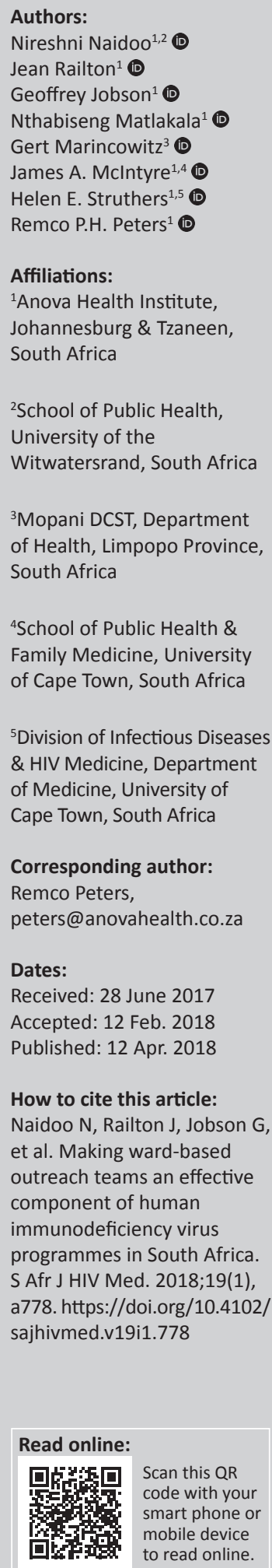

${ }^{4}$ School of Public Health \& Family Medicine, University of Cape Town, South Africa

${ }^{5}$ Division of Infectious Diseases \& HIV Medicine, Department of Medicine, University of Cape Town, South Africa

\section{Corresponding author:} Remco Peters, peters@anovahealth.co.za

\section{Dates:}

Received: 28 June 2017 Accepted: 12 Feb. 2018 Published: 12 Apr. 2018

How to cite this article: Naidoo N, Railton J, Jobson G, et al. Making ward-based outreach teams an effective component of human immunodeficiency virus programmes in South Africa. S Afr J HIV Med. 2018;19(1) a778. https://doi.org/10.4102/ sajhivmed.v19i1.778

Read online: Scan this QR
code with your
smart phone or
mobile device
to read online.

\begin{abstract}
The implementation of ward-based outreach teams (WBOTs), comprised of community health workers (CHWs), is one of the three interventions of the South African National Department of Health's (NDoH) Primary Health Care (PHC) Re-engineering strategy for improving health outcomes. CHWs provide a necessary structure to contribute to successful implementation of the human immunodeficiency virus (HIV) programme in four ways: (1) prevention of HIV infection by health education, (2) linkage to care by health education and referrals, (3) adherence support and (4) identification of individuals who are failing treatment. However, CHW programme and HIV programme-specific barriers exist that need to be resolved in order to achieve maximum impact. These include a lack of stakeholder and community support for WBOTs, challenging work and operational environments, a lack of in-depth knowledge and skills, and socio-cultural barriers such as HIV-related stigma. Considering its promising structure, documentation of the WBOT contribution to healthcare overall, and the HIV programme in particular, is urgently warranted to successfully and sustainably incorporate it into the South African healthcare system.
\end{abstract}

\section{Introduction}

Ward-based outreach teams (WBOTs) are becoming an increasingly important part of primary healthcare (PHC) in resource-constrained rural settings across the globe. ${ }^{1}$ In addition to district clinical specialist teams and integrated school health services, WBOTs are one of the three components of the PHC re-engineering programme introduced in South Africa in 2012 to improve communities' access to health services and the quality of care provided. ${ }^{2}$ In South Africa, each WBOT is linked to a PHC facility and consists of a team leader, usually a professional nurse, plus five or more community health workers (CHWs). Each CHW is allocated 250-400 households to support. ${ }^{3}$

Several approaches have been initiated to improve outcomes of the HIV programme in South Africa, including human immunodeficiency virus (HIV) counselling and testing and adherence clubs. ${ }^{4}$ Specifically, these approaches have been either community-based or facility-based. WBOTs provide a means of bridging the gap between health facilities and the communities they serve, a role which may be especially valuable in rural areas where communities are impoverished and under-served, and where individuals have to travel excessive distances to seek medical care. ${ }^{1}$ CHW's primary activities include registration of household's health needs, provision of health education and the provision of adherence support for individuals on antiretroviral therapy (ART) or with other chronic illnesses. In this article, we identify and discuss key opportunities for effectively integrating WBOTs in the HIV response in South Africa, while noting various challenges that need to be addressed in order to maximise impact.

\section{Key opportunities for ward-based outreach teams in South Africa's human immunodeficiency virus programme}

The WBOTs are well positioned at the interface of the community and facility to contribute to the HIV programme in the following ways (Table 1):

- provision of information to educate individuals on how to prevent transmission of HIV infection

- identification of individuals at risk for HIV infection who should be tested for HIV 
TABLE 1: Summary of potential contributions of WBOTs to the HIV programme

\begin{tabular}{|c|c|c|}
\hline Opportunities & Activities of WBOTs & Expected outcomes \\
\hline $\begin{array}{l}\text { 1. Provision of information } \\
\text { to educate individuals } \\
\text { on how to prevent } \\
\text { transmission of HIV } \\
\text { infection }\end{array}$ & $\begin{array}{l}\text { - Educate community } \\
\text { about HIV prevention } \\
\text { during household visits }\end{array}$ & $\begin{array}{l}\text { - Reduction in newly } \\
\text { HIV-infected individuals }\end{array}$ \\
\hline $\begin{array}{l}\text { 2. Identification of } \\
\text { individuals at risk for } \\
\text { HIV infection who } \\
\text { should be tested } \\
\text { for HIV }\end{array}$ & $\begin{array}{l}\text { - Re-educate individuals } \\
\text { about HIV } \\
\text { - Refer individuals to the } \\
\text { PHC facility for HIV } \\
\text { testing }\end{array}$ & $\begin{array}{l}\text { - Increased uptake of HIV } \\
\text { testing and ART initiation }\end{array}$ \\
\hline $\begin{array}{l}\text { 3. Provision of adherence } \\
\text { support and tracing of } \\
\text { individuals with missed } \\
\text { appointments to } \\
\text { improve retention } \\
\text { in care }\end{array}$ & $\begin{array}{l}\text { - Refer defaulting } \\
\text { individuals back to the } \\
\text { PHC facilities } \\
\text { - Implement adherence } \\
\text { health clubs to support } \\
\text { ART maintenance for } \\
\text { groups of HIV-infected } \\
\text { stable individuals }\end{array}$ & $\begin{array}{l}\text { - Reduction in number of } \\
\text { individuals defaulting in } \\
\text { treatment taking }\end{array}$ \\
\hline $\begin{array}{l}\text { 4. Early identification of } \\
\text { individuals with } \\
\text { deteriorating health } \\
\text { while on ART to reduce } \\
\text { further morbidity and } \\
\text { mortality }\end{array}$ & $\begin{array}{l}\text { - Refer individuals with } \\
\text { deteriorating health due } \\
\text { to chronic diseases to the } \\
\text { clinic for treatment } \\
\text { - Distribute medication to } \\
\text { bedridden patients }\end{array}$ & $\begin{array}{l}\text { - Reduction in mortality } \\
\text { and morbidity of } \\
\text { HIV-infected individuals } \\
\text { with deteriorating health } \\
\text { and chronic diseases }\end{array}$ \\
\hline
\end{tabular}

WBOT, ward-based outreach teams.

- provision of adherence support and tracing of individuals with missed appointments to improve retention in care

- early identification of individuals with deteriorating health while on ART to reduce further morbidity and mortality.

\section{Prevention of human immunodeficiency virus infection and its complications}

There is evidence showing the effectiveness of using CHWs in large-scale health promotion and disease prevention programmes in low-income countries such as Haiti, Ethiopia, Uganda and Malawi. ${ }^{5,6}$ This preventative aspect of CHWs' work could also play a critical role in South Africa's HIV programme. CHWs could support the programme specifically not only by providing health education on how to prevent HIV infection but also by distributing condoms and potentially assisting in the identification of individuals that may benefit from pre-exposure prophylaxis (PrEP). ${ }^{7,8,9,10}$ CHWs, through adherence support, could contribute to lowering community viral load (VL) and thereby reducing transmission in the community. ${ }^{11,12}$ However, it has not yet been documented how well the above roles are performed and whether $\mathrm{CHWs}^{\prime}$ efforts positively impact on the reduction of HIV incidence. To our knowledge, there are so far no South African studies undertaken that assess the impact of preventative aspect of CHWs' work.

\section{Identification of human immunodeficiency virus-infected individuals}

CHW's link between communities and facilities makes them well placed to support and appropriately refer vulnerable individuals to health facilities. Amongst those classified as vulnerable by the National Department of Health $(\mathrm{NDoH})$ are individuals at risk for HIV infection and those already infected. ${ }^{3}$ As such, discussion of HIV status and HIV risk with individuals, their partners and children is an important component of CHW activity. South Africa is one of the first countries to formally adopt the Universal Test and Treat approach recommended in the updated
World Health Organization (WHO) guidelines on HIV treatment. The guidelines state that all HIV-infected individuals should start ART within two weeks of an initial CD4 count being performed. ${ }^{13}$ There are limited data about the feasibility of HIV case identification by CHWs in South Africa, but a study by Uys, conducted at seven sites in South Africa, shows that community caregivers who provide psychosocial and household support may have a positive impact on HIV case identification through mobilisation and referral of individuals to HIV services for testing. ${ }^{14} \mathrm{CHWs}$, on the contrary, focus on providing medical aspects of care. Although this study was conducted in the setting of a homebased AIDS care model, and community caregivers are not the same as $\mathrm{CHWs}$, the results are encouraging and support the involvement of a structure such as WBOT that bridges the gap between the community and the healthcare facility by complementing services provided by community caregivers. $^{15}$

CHWs could also make an important contribution to the HIV response by supporting home-based HIV testing. This approach to HIV testing and counselling was used in a cluster randomised controlled trial in KwaZulu-Natal Province, South Africa, which found significantly higher rates of HIV testing amongst participants in the home-based testing arm than those testing at facilities (69\% vs. $47 \%$; $95 \%$ CI $1.32-1.81$ ). Other results included a higher uptake of couple counselling and testing as well as a reduction in multiple partners. ${ }^{16}$ In light of this, to strengthen the impact of CHWs in the HIV programme, home-based testing by CHWs should be considered. There is also potential for HIV testing during

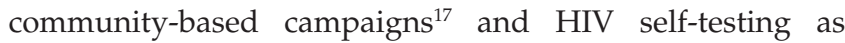
recommended by the WHO. ${ }^{18}$

\section{Tracing non-adherent individuals and provision of adherence support to improve retention in care}

A high level of patient retention on antiretroviral treatment is imperative for good HIV programme outcomes. CHWs can improve retention in care directly by tracing individuals who missed their appointments or defaulted treatment and linking them back into care at the healthcare facility. Although tracing is an important part of the CHW's activities, the effectiveness of this approach remains undocumented. Defaulter tracing should not become so time consuming that it prevents $\mathrm{CHWs}$ from effectively undertaking their other duties. In the South African WBOT programme, we suggest that defaulter tracing should combine an initial attempt to contact non-adherent individuals telephonically, followed by physical tracing at their homes by CHWs if they are not reachable via telephone. This was shown to be successful in other studies ${ }^{19}$, and preliminary data from Mopani District, Limpopo Province, are promising, suggesting that there is value in using a combination of telephone and physical tracing as a routine CHW activity (R.P. Peters, 2017, n.d., personal communication).

Another way in which CHWs could contribute to improving retention in care is through adherence support. Grimwood 
et $a l .{ }^{20}$ in a multicentre cohort study, demonstrated that community-based adherence support is an effective way to improve patient retention on ART. In that study, patient advocates (PAs) were tasked with providing adherence support to the community. Clinical, virological and immunological outcomes were compared between children who did and did not receive community-based adherence support from PAs in Western Cape, KwaZulu-Natal, Eastern Cape and Mpumalanga provinces. They showed a significant difference in patient retention after three years: $92 \%$ (95\% CI: $76.7 \%-97.6 \%)$ amongst children with PAs and 74\% (95\% CI: $65.4 \%-81.0 \%$ ) amongst children without PA support. In addition, multivariable analyses showed that children with PAs had reduced probability of HIV-related programme attrition and mortality. ${ }^{20}$ Similarly, Igumbor et al. ${ }^{12}$ found that a significantly higher proportion of patients (70\% vs. $30 \%$ ) with a community-based adherence supporter had a VL of less than 400 copies/mL at six months of treatment. ${ }^{12}$ In the context of the WBOT programme, adherence support entails regularly visiting patient's households to supervise medication taking, educating patients about the importance of taking treatment exactly as prescribed by the healthcare workers, provision of tips and tools such as diary record cards to aid in timely treatment taking and referrals of non-adherent individuals to the nearest PHC facility for follow-up.

The positive value of adherence support in retaining individuals on ART in South Africa was also documented in the study by Igumbor et al., ${ }^{12}$ which evaluated the effect of community adherence support on ART programme retention. They observed that a significantly higher proportion of patients with a community-based adherence supporter (89\%) had a treatment pick-up rate of over 95\%. ${ }^{12}$ Adherence support by CHWs may also play an important role in reducing health facility workloads through monitoring and supporting HIV-infected patients who are stable on ART. 21,22,23 Grimsrud et al., ${ }^{4}$ for example, found that task-shifting to CHWs can successfully support and improve ART adherence and encourage patient self-management. ${ }^{4}$ Over a period of 12 months, more than 2000 stable ART patients were successfully decentralised from a doctor-driven PHC clinic to a community-based model of care including $\mathrm{CHWs}$, while maintaining ART retention rates of $97 \%$ after six months and $94 \%$ at 12 months. ${ }^{4}$ The ongoing scale-up of the ART programme in South Africa, particularly as test and treat becomes routine, makes it increasingly important to find effective ways to involve CHWs in novel systems of alternative distribution of ART and long-term ART retention, especially in resource-limited settings.

\section{Early identification of individuals failing treatment}

Finally, CHWs have a critical role to play in maintaining HIV viral suppression and hence reducing HIV-related morbidity and mortality through ongoing monitoring of individuals on ART. In this role, CHWs essentially provide a means for the early detection of individuals at risk of defaulting or treatment failure through symptom screening. By enhancing treatment literacy, early identification of ART side effects, awareness of psychosocial and poverty-related factors that may impact the likelihood of patient's virological failure, CHWs can be an essential link in the HIV treatment cascade. Grimwood et al..$^{20}$ demonstrate this potential by finding that the presence of community-based patient support reduced mortality of children after three years of ART: $3.7 \%$ of children died in a setting with PAs, whereas $8.0 \%$ of children died without community-based support. ${ }^{20}$

\section{Barriers and challenges to successful ward-based outreach team support}

Despite the promise of the WBOTs in improving programme outcomes, there are several key barriers and challenges to their successful implementation in South Africa's healthcare system. These include general operational challenges and HIV programme-specific challenges as discussed below.

\section{General challenges}

\section{Varying perceptions of community health worker roles}

The health system identifies CHWs as volunteers who manage patients within the community in which they reside and usually receive a stipend for their services. ${ }^{24}$ Some community members perceive $\mathrm{CHWs}$ to be peers, while others are knowledgeable about $\mathrm{CHW}$ roles in communities such as promoting primary health services and encouraging testing for several health conditions. ${ }^{25} \mathrm{CHWs}$ generally perceive themselves as being a part of the community, while providing services and making a difference to the lives of people. Some even have an elevated perception of their roles as being teachers, social workers and doctors as they are given access to privileged medical information of patients and are linked to the formal health system. ${ }^{26}$ However, this empowerment may be challenged by difficult working environments. A recent multi-country comparative study shows that CHWs are often left feeling unsupported and undervalued as organisational and relational challenges hindered their work. Therefore, improving organisational support and helping the community better understand and appreciate the roles of CHWs could possibly mitigate these challenges faced by $\mathrm{CHWs} .^{27}$

\section{Lack of stakeholder and community support}

A fundamental challenge that limits the effectiveness of WBOTs in South Africa is their poor integration into local health systems, which in turn undermines the role of CHWs in community healthcare. ${ }^{7}$ Effective integration of WBOTs requires $\mathrm{PHC}$ facilities in the $\mathrm{CHW}$ programme to adopt a coordinating role ${ }^{28}$ and to facilitate ongoing contact with CHWs for the purposes of feedback, training and monitoring. A study in Uganda, for example, found that creating a bidirectional feedback loop by holding monthly meetings and using data collected timeously improved $\mathrm{CHW}$ activities 
and ensured that the continuum of services from community to facility is maintained. ${ }^{29}$ The integration of CHWs into a well-established community-based PHC system with facility nurses, doctors, a proper referral system and adequate financing and resources is essential in relieving pressures of health service delivery. ${ }^{1,30}$

In addition, support from community leaders and social workers as well as community acceptance plays an important role in the success of the $\mathrm{CHW}$ programme. Evaluating the interaction of CHWs and the community from a cultural and gender perspective is therefore imperative; for example, male community members may resist interaction with female CHWs. ${ }^{31}$ Gender inequalities have also been shown to negatively impact HIV counselling and testing, HIV status disclosure and ART adherence, as well as the ability of female CHWs to work with male community members. ${ }^{32}$ In this regard, the introduction of male CHWs may be necessary to address these gender-related challenges and may result in improvement in health outcomes of male community members. ${ }^{33}$ However, the introduction of male CHWs may be thwarted by existing norms of hegemonic masculinity, where male CHWs assert dominance over their clients, limiting male community member's ability to be open about being sick and vulnerable. ${ }^{34}$ This could also result in female community members feeling intimidated and withdrawn. When making these decisions to introduce male CHWs into the programme, it is imperative to adopt an approach that is conducive to developing supportive relationships between $\mathrm{CHWs}$ and community members that improve health-seeking behaviour. ${ }^{34}$

\section{Challenging work environments and operational barriers}

Minimal, inconsistent or irregular payment of stipends remains a critical challenge in maintaining $\mathrm{CHWs}$ interest and involvement in community-based programmes.,28 Common reasons for $\mathrm{CHWs}$ resigning include inadequate compensation for their time, and family members being unsupportive of their workload in light of the minimal incentive received. ${ }^{23,35}$ Limited practical resources can also inhibit the effectiveness of CHW programmes. For example, a lack of comfortable footwear and umbrellas to protect CHWs from harsh weather elements may prohibit them from conducting household visits. The fact the CHWs have not been provided with official uniforms or name tags may also present a problem with regard to identification and authority of the CHWs in the community and health systems. Funding constraints also mean that CHWs often lack resources to travel to households, meetings and training sessions. ${ }^{28}$ Evaluations of CHW programmes show that CHWs become overwhelmed by their broad range of tasks, resulting in poor performance and work overload. Hermann et al. ${ }^{6}$ note that CHWs with a large number of tasks tend to select a few that they perceive as the most important or feasible to prioritise. ${ }^{6}$ The tasks they prioritise may or may not align with programmatic goals. Therefore, clearly defined management and reporting channels are essential to ensure that WBOTs are effectively used in the South African HIV programme. Moreover, the use of mobile technology should be considered to assist CHWs with their tasks and to improve communication. Studies show that the use of mobile technology by CHWs to collect field-based health data, receive reminders and alerts, facilitate health education and conduct person-to-person communication improved quality of care, service efficiency and programme monitoring. ${ }^{36,37}$

\section{Human immunodeficiency virus programme challenges}

\section{Lack of knowledge and skills}

In South Africa, the $\mathrm{NDoH}$ developed a curriculum for CHWs, with the ultimate goal of national certification. ${ }^{38}$ CHWs are currently trained on HIV prevention and treatment, including provision of health education, screening community members for health problems and referrals to local facilities, included in the phase one training. They are therefore expected to have adequate knowledge to do their work. However, sustaining and updating knowledge through continual training is a challenge.$^{28}$ Continuous education and support is therefore a requirement to sustain quality of the programme. Phase two training is currently underway, which will address knowledge gaps. ${ }^{38}$

\section{Socio-cultural barriers}

In a context where HIV is stigmatised, CHWs may be seen as a marker of HIV or AIDS as they are known to support individuals who are HIV-positive. This, in turn, can arouse a feeling of stigmatisation in community members which may result in individuals refusing to talk to CHWs, providing incorrect contact information, pretending to be someone else when visited by $\mathrm{CHWs}$ and asking to change the location of $\mathrm{CHW}$ visits. For example, there has been a report of CHWs specifically providing ART adherence support, trying to conceal their identities when visiting households by refraining from wearing uniforms given to them by their NGOs and pretending to be selling various commodities. ${ }^{31}$ Implementing community awareness and education campaigns aimed at reducing HIV-related stigma may therefore be an important factor facilitating the effectiveness of WBOTs. This may enable individuals to feel more comfortable to provide accurate medical information to their $\mathrm{CHWs}$ and details regarding treatment options can be shared with them by CHWs. This will also enable individuals to build relationships with external sources of support such as CHWs and PHC facilities in order to obtain healthcare advice and treatment. ${ }^{31} \mathrm{~A}$ formal WBOT policy is still in the process of being finalised. The policy may include ethical guidelines governing their roles, such as the requirement of registration with health bodies (currently not required), ethics training and the legal framework in which the CHWs operate. ${ }^{38}$ The policy may also stipulate the extent to which CHWs are qualified to provide health advice. This may improve $\mathrm{CHWs}$ credibility in the community.

\section{Perspective}

There is great potential for CHWs to have a substantial impact on HIV programmes as evidenced above. We have presented 
several examples of successful CHW initiatives. CHW efforts can lead to improved health outcomes by improving community access to PHC in terms of health education, patient linkage to and retention in care. Expanding the $\mathrm{CHW}$ programme will further increase the potential to address large healthcare gaps. However, health system barriers such as human resource capacity, inconsistent remuneration, lack of nationally recognised training and a failure to mainstream WBOTs are important factors that limit the effectiveness of the WBOT programme. These need to be addressed for WBOTs to have maximum impact on the HIV and other health programmes. ${ }^{23} \mathrm{~A}$ particular point of concern is that $\mathrm{CHWs}$ may be overloaded with expectations and activities. In that regard, it is essential that the workforce size and quality of services provided must match the programme deliverables. Further investment in the WBOT programme would be essential to expand it; however, this is not easily achieved without a broadscale documented impact. To our knowledge, there are no comprehensive data available on the impact and contribution of the WBOT structure on HIV programme outcomes in South Africa. Such impact would likely be highest in rural settings with high poverty and low education rates, but at the same time the operational barriers may be larger in those areas. ${ }^{23}$ Several initiatives are currently underway to document the impact that CHWs have on health programmes in general, and the HIV programme in particular, as well as to assess practical ways to improve their contribution. Community healthcare provides an important component of the South African healthcare system with high promise to support the usually overcrowded healthcare facilities, but strengthening of the structure is warranted to obtain maximum impact.

\section{Conclusion}

There is evidence that WBOTs have a positive impact on healthcare. However, despite the strong potential, there are limited data on the impact of WBOTs on the South African HIV programme. Therefore, evaluations to determine operational barriers and opportunities for improvement are urgently warranted. This will provide much needed information to stakeholders regarding areas that need improvement and strengthening in order for WBOTs to become a sustainable structure in the South African healthcare system.

\section{Acknowledgements}

Division of Epidemiology and Biostatistics, School of Public Health, Faculty of Health Sciences, University of the Witwatersrand, Johannesburg, South Africa. This research project was supported by a postgraduate training scholarship from the Fogarty and NIAID, The UNC-Wits AIDS Implementation Science and Cohort Analyses Training Grant (Grant number: 5D43TW009774-02). The content is solely the responsibility of the authors and does not necessarily represent the official views of the Fogarty and NIAID.

Anova Health Institute is supported by the US President's Emergency Plan for AIDS Relief (PEPFAR) programme via the US Agency for International Development (USAID) under Cooperative Agreement No. AID-674-A-12-00028. The views expressed in this manuscript do not necessarily reflect those of PEPFAR or USAID.

\section{Competing interests}

The authors declare that they have no financial or personal relationships which may have inappropriately influenced them in writing this article.

\section{Authors' contributions}

N.N. conceptualised the study and undertook the writing of the manuscript. J.R., G.J., N.M., G.M., J.A.M. and H.E.S. contributed to the manuscript review. R.P.H.P. conceptualised the study and contributed to the in-depth review of the manuscript.

\section{References}

1. Lui A, Sullivan S, Khan M, Sachs S, Singh P. Community health workers in global health: Scale and scalability. Mt Sinai J Med. 2011;78:419-435. https://doi org/10.1002/msj.20260

2. Pillay $Y$, Barron P. The implementation of PHC re-engineering in South Africa [homepage on the Internet]. Public Health Association of South Africa; 2012 [cited 2017 Mar 10]. Available from: http://www.phasa.org.za/articles

3. Naledi T Baron P, Schneider H. Primary Health Care in SA since 1994 and implications of the new vision for PHC re-engineering. SAHR. Durban: Health Systems Trust; 2011; p. 17-28.

4. Grimsrud A, Sharp J, Kalombo C, Bekker L, Myer L. Implementation of communitybased adherence clubs for stable antiretroviral therapy patients in Cape Town, South Africa. JIAS. 2015;18:19984. https://doi.org/10.7448/IAS.18.1.19984

5. Mukherjee JS, Eustache FRE. Community health workers as a cornerstone for integrating HIV and primary healthcare. AIDS Care. 2007;19(1):73-82.

6. Hermann K, van Damme W, Pariyo GW, Schouten E, Assefa Y, Cirera AWM Community health workers for ART in sub-Saharan Africa: Learning from experience - Capitalizing on new opportunities. Hum Resour Health 2009;7:31. https://doi.org/10.1186/1478-4491-7-31

7. Schneider $\mathrm{H}$, Hlope $H$, van Rensburg D. Community health workers and response to HIV/AIDS in South Africa: Tensions and prospects. Health Pol Plan. 2008;23(3):179-187. https://doi.org/10.1093/heapol/czn006

8. Baral S, Beyrer C, Muessig K, et al. Burden of HIV among female sex workers in low-income and middle-income countries: A systematic review and meta-analysis.
Lancet Infect Dis. 2012;12(7):538-549. https://doi.org/10.1016/S1473-3099(12) 70066-X

9. Beyrer C, Baral SD, van Griensven F, et al. Global epidemiology of HIV infection in men who have sex with men. Lancet. 2012;380(9839):367-377. https://doi.org/ 10.1016/S0140-6736(12)60821-6

10. Bekker L-G, Rebe K, Venter F, et al. Southern African guidelines on the safe use of pre-exposure prophylaxis in persons at risk of acquiring HIV-1 infection. S Afr J HIV Med 2016;17:a455.

11. Chang LW, Kagaayi J, Nakigozi G, et al. Effect of peer health workers on AIDS care in Rakai, Uganda: A cluster-randomized trial. PLoS One. 2010;5(6):e10923. https://doi.org/10.1371/journal.pone.0010923

12. Igumbor JO, Scheepers E, Ebrahim R, Jason A, Grimwood A. An evaluation of the impact of a community-based adherence support programme on ART outcomes in selected government HIV treatment sites in South Africa. AIDS Care. 2011;23(2):231-236. https://doi.org/10.1080/09540121.2010.498909

13. NDoH. Implementation of the universal test and treat strategy for HIV positive patients and differentiated care for stable patients [homepage on the Internet]. National Department of Health; 2016 [cited 2017 Mar 29]. Available from: http:// www.sahivsoc.org/Files/22\%208\%2016\%20Circular $\% 20$ UTT $\% 20 \% 20 \% 20$ Decongestion\%20CCMT\%20Directorate\%20(2).pdf

14. Uys LR. The practice of community caregivers in a home-based HIV/AIDS project in South Africa. J Clin Nurs. 2002;11(1):99-108. https://doi.org/10.1046/j.13652702.2002.00566.x

15. Sips I, Haeri Mazanderani A, Schneider H, Greeff M, Barten F, Moshabela M. Community care workers, poor referral networks and consumption of personal resources in rural South Africa. PLoS One. 2014;9(4):e95324. https://doi. org/10.1371/journal.pone.0095324

16. Doherty T, Tabana H, Jackson D, et al. Effect of home based HIV counselling and testing intervention in rural South Africa: Cluster randomised trial. BMJ. 2013;346:f3481. https://doi.org/10.1136/bmj.f3481

17. Reif LK, Rivera V, Louis B, et al. Community-based HIV and health testing for highrisk adolescents and youth. AIDS Patient Care STDs. 2016;30(8):371-378. https:// doi.org/10.1089/apc.2016.0102

18. WHO. WHO recommends HIV self-testing [homepage on the Internet]. World Health Organization; 2016 [cited 2017 Mar 29]. Available from: http://www.who. int/hiv/pub/vct/alternate_Policy-brief_HIVST-Asia.pdf?ua=1 
19. Allen CG, Escoffery C, Satsangi A, Brownstein JN. Strategies to improve the integration of community health workers into health care teams: 'A little fish in a integration of community health workers into health care teams: 'A little fish in a
big pond'. Prev Chronic Dis. 2015;12:E154. https://doi.org/10.5888/pcd12.150199

20. Grimwood A, Fatti G, Mothibi E, Malahela M, Shea J, Eley B. Community adherence support improves programme retention in children on antiretroviral treatment: $A$ multicentre cohort study in South Africa. J Int AIDS Soc. 2012;15:17381. https:// doi.org/10.7448/IAS.15.2.17381

21. le Roux K, le Roux I, Mbewu N, Davis E. The role of community health workers in the re-engineering of primary health care in Rural Eastern Cape. S Afr Fam Pract. 2015;57(2):116-120. https://doi.org/10.1080/20786190.2014.977063

22. Cohen $\mathrm{R}$, Lynch $\mathrm{S}$, Bygrave $\mathrm{H}$, et al. Antiretroviral treatment outcomes from nurse-driven community supported HIV/AIDS treatment programme in rura Lesotho: Observational cohort assessment at two years. J Int AIDS Soc. 2009;12:23. https://doi.org/10.1186/1758-2652-12-23

23. Mwai GW, Mburu G, Torpey K, Frost P, Ford N, Seeley J. Role and outcomes of community health workers in HIV care in sub-Saharan Africa: A systematic review. J Int AIDS Soc. 2013;16:18586. https://doi.org/10.7448/IAS.16.1.18586

24. Tsolekile LP, Puoane T, Schneider H, Levitt NS, Steyn K. The roles of community health workers in management of non-communicable diseases in an urban township. Afr J Prim Health Care Fam Med. 2014;6(1):E1-E8.

25. Rachlis B, Naanyu V, Wachira J, et al. Community Perceptions of Community Health Workers (CHWs) and their roles in management for HIV, tuberculosis and hypertension in western Kenya. PLoS One. 2016;11(2):e0149412. https://doi. org/10.1371/journal.pone.0149412

26. Mlotshwa L, Harris B, Schneider H, Moshabela M. Exploring the perceptions and experiences of community health workers using role identity theory. Glob Health Action. 2015;8:28045. https://doi.org/10.3402/gha.v8.28045

27. Naimoli JF, Frymus DE, Wuliji T, Franco LM, Newsome MH. A community health worker 'logic model': Towards a theory of enhanced performance in low- and middle-income countries. Hum Res Health. 2014;12:56. https://doi.org/10.1186/ 1478-4491-12-56

28. Horwood CM, Youngleson EM, Stern AF, Barker PM. Using adapted qualityimprovement approaches to strengthen community-based health systems and improve care in high HIV-burden sub-Saharan African countries. AIDS. 2015;29 (Suppl 2):S155-S164. https://doi.org/10.1097/QAD.0000000000000716
29. Wools-Kaloustian KK, Sidle JE, Selke HM, et al. A model for extending antiretroviral care beyond the rural health centre. J Int AIDS Soc. 2009;12:22. https://doi.org/ 10.1186/1758-2652-12-22

30. Bajpai N, Dholakai R. Improving the performance of Accredited Social Health Activists (ASHAs) in India. New Delhi: International Advisory Panel of the National Rura Health Mission, Ministry of Health and Family Welfare, Government of India; 2011.

31. Masquillier C, Wouters E, Mortelmans D, van Wyk B, Hausler H, Van Damme W. HIV/AIDS competent households: Interaction between a health-enabling environment and community-based treatment adherence support for people living with HIV/AIDS in South Africa. PLoS One. 2016;11(3):e0151379. https://doi. org/10.1371/journal.pone.0151379

32. Treffry-Goatley A, Lessells $R$, Sykes $P$, et al. Understanding specific contexts of antiretroviral therapy adherence in rural South Africa: A thematic analysis of digital stories from a community with high HIV prevalence. PLoS One. 2016;11(2):e0148801. https://doi.org/10.1371/journal.pone.0148801

33. Martinez Perez G, Cox V, Ellman T, et al. 'I know that I do have HIV but nobody saw me': Oral HIV self-testing in an informal settlement in South Africa. PLoS One. 2016;11(4):e0152653. https://doi.org/10.1371/journal.pone.0152653

34. Gittings L. 'When you visit a man you should prepare yourself': Male community care worker approaches to working with men living with HIV in Cape Town, South Africa. Cult Health Sex. 2016;18(8):936-950. https://doi.org/10.1080/13691058. 2016.1150513

35. UNICEF. What works for children in South Asia, community health workers [homepage on the Internet]. Regional Office for South Asia: The United Nations Children's Fund; 2004 [cited 2016 May 18]. Available from: http://www.unicef. org/rosa/community.pdf

36. Braun R, Catalani C, Wimbush J, Israelski D. Community health workers and mobile technology: A systematic review of the literature. PLoS One. 2013;8(6):e65772. https://doi.org/10.1371/journal.pone.0065772

37. Schuttner L, Sindano N, Theis M, et al. A mobile phone-based, community health worker program for referral, follow-up, and service outreach in rural Zambia:
Outcomes and overview. Telemed J E Health. 2014;20(8):721-728. https://doi. org/10.1089/tmj.2013.0240

38. National Department of Health. National health insurance for South Africa (White paper). Pretoria: NDOH; 2015 